\title{
Selection of critical events in nuclear fragmentation
}

\author{
C. O. Dorso ${ }^{1}$ and J. A. López ${ }^{2}$ \\ ${ }^{1}$ Universidad de Buenos Aires, Nuñez, 1428, Buenos Aires, Argentina \\ ${ }^{2}$ University of Texas at El Paso, El Paso, Texas 79968 \\ (Received 27 March 2001; published 23 July 2001)
}

\begin{abstract}
The calculation of the critical exponent $\tau$ is crucial in the determination of critical phenomena in heavy ion reactions. This, however, is obscured by the unavoidable mixing of critical and noncritical events that results in nonclean signals. Here we report on a method to extract critical events from a set of mixed ones. In comparing to the traditional one, based on the so-called Campi plot, a distinct advantage is found.
\end{abstract}

DOI: 10.1103/PhysRevC.64.027602

PACS number(s): 25.70.Pq, 24.10.Lx, 25.70.Mn, 65.20.+w

Since the pioneering work of the Purdue group, in which the mass spectra resulting from multifragmentation of excited nuclei was first fitted to a power law, a lot of work has been devoted to the characterization of this phenomenon as a phase transition. This task has been undertaken both theoretically and experimentally (for a recent review see Refs. [1,2]) via the calculation of critical exponents [3], caloric curves $[4,5]$, thermal response functions [6-9], etc.

In particular, the critical exponent $\tau$ can be extracted from the mass spectrum of events produced exactly at the critical point, which should have a power-law decay such as $n_{A}$ $\approx A^{-\tau}$, where $n_{A}$ is the number of fragments of size $A$ per unit mass. At nearby points, the yield is modified to $n_{A}$ $\approx A^{-\tau} f(z)$ by a scaling factor $f(z)$ which obscures the pure power law. In the scaling factor $z=A^{\sigma} \varepsilon, \varepsilon$ is the relative distance to the critical point $\varepsilon=\left(p-p_{c}\right) / p_{c}$, and $\sigma$ is another critical exponent. As the experimental data contains an assortment of events at different impact parameters, excitation energies, etc., the resulting spectra will unavoidable contain a mix of critical and noncritical events.

Originally, the critical exponent was estimated from mixed data by means of a fitting parameter ("apparent exponent") resulting from a power-law fit to critical and noncritical data. Since the scaling function attains the value of 1 at the critical point $f(0)=1$, the minimum of this apparent exponent was identified with the true critical exponent, where a pure power law would apply (see Ref. [1]). Nowadays, whenever possible, critical events must extracted from the data and used to calculate $\tau$ directly from them. Unfortunately, the most commonly used technique that presumably selects critical and near-critical events, based on the socalled Campi plot $[7,8,10]$, does not live up to its promises.

A Campi plot is a representation of data from multiple events in which the log of the size of the maximum fragment in event $i \ln \left(A_{\max }^{i}\right)$ is plotted against the logarithm of the second moment of the mass distribution in the same event $\ln \left(M_{2}^{i}\right)$. (The $k$ th moment is defined as $M_{k}=\sum A^{k} n_{A}$, with the biggest fragment excluded from the sum in the "liquid side" [11].) The resulting graph displays a typical "boomerang" shape with a down-pointing branch believed to be associated to overcritical events (i.e., an exponential mass yield with large multiplicity and small biggest fragment), an horizontal branch presumably related to undercritical events (i.e., a "U-shaped" distribution of large biggest cluster and small multiplicity), and an "elbow" region usually taken as connected to critical events with power-law mass distributions [12].

In previous works, rather ad libitum criteria have been used to select events, presumably critical, from the elbow region and to extract $\tau$ from them [12]. Unfortunately, this results in a wide mix of noncritical and critical events. We now use percolation to critically examine the effects of this criterion on the resulting value of the critical exponent.

Bond percolation is a simple procedure that displays critical behavior [11,13-17]. A three-dimensional grid (of say, $L \times L \times L$ nodes) can be fragmented into connected segments by breaking each of the internode links with some probability $p_{b}$. Repeating this process for many grids and combining the results, produces a smooth distribution of "masses," i.e., number of connected nodes. At some critical bond breaking probability $p_{c}(L)$, the resulting mass distribution follows a characteristic power-law decay, but mixing results from different $p_{c}$ 's produces a scaled power-law decay different from the critical one. Critical and noncritical events tend to populate different, but overlapping, segments of the Campi plot.

We now examine the wide mixing of critical and noncritical events produced by the ad libitum criteria used in the past to select events from the elbow region. This will be done using an $L=6$ grid which will be broken 20000 times with each of the probabilities $p=0.01,0.02, \ldots, 0.99$ for a total of almost 2 million events. Using the resulting mass spectra a Campi plot will be populated, and a subset of events will be selected from the elbow region, and used to obtain a critical exponent.

The critical bond activation probability $p_{c}(L)$, and the corresponding exponent $\tau$ can be calculated as explained by Elliot et al. [3]. The critical exponent must be obtained from a one-parameter fit $(\tau)$ as the overall normalization $q_{0}$ is determined via the Riemann $\zeta$ function $q_{0}=1 / \Sigma_{A} A^{1-\tau}$, with the mass distribution $n_{A}$ normalized to the system size and $M_{1}$ to 1 .

The critical mass spectra is selected as that best fitted by this single-parameter power law according to a minimum $\chi^{2}$. This fitting procedures are performed in the mass range between $0.02 A_{\text {tot }}$ and $0.15 A_{\text {tot }}$ to avoid finite size effects. The bond probability value for which the best fit occurs is 


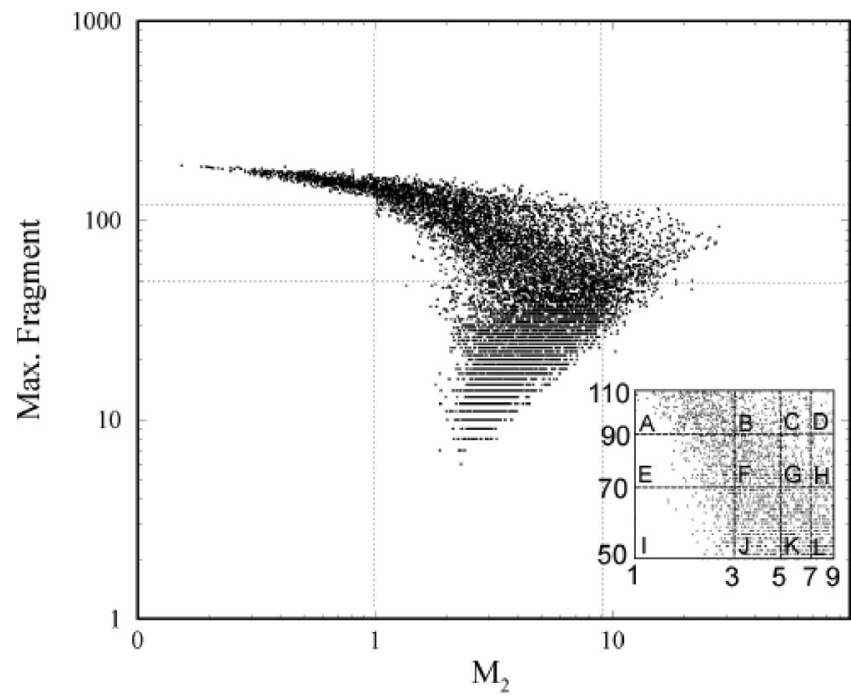

FIG. 1. Campi plot of percolation data produced with almost 2 million breakups of a $6 \times 6 \times 6$ grid at several breakup probabilities. The central rectangular region shows the cut used to select nearcritical events. The inset shows the subdivision of the central region used in the text.

$p_{c}(6) \cong 0.32 \pm 0.01$ and the corresponding critical exponent is $\tau \cong 2.19 \pm 0.02$, both quite consistent with previous calculations.

We now proceed to study the effects of the event selector. The Campi plot resulting from the percolation data is shown in Fig. 1 along with a rectangular region used as an event selector in the references mentioned before. The left panel of Fig. 2 shows the mass distribution of the events contained in the central rectangle. The corresponding critical exponent is $\tau=2.3 \pm 0.02$, quite a distance away from the true value calculated before. The origin of this discrepancy can be found by inspecting the bond probability distribution of the events selected by the cut.

The bond probability distribution can defined as $P_{i}$ $=n\left(p_{i}\right) / \Sigma_{i} n\left(p_{i}\right)$ with $n\left(p_{i}\right)$ representing the number of events with $p_{b}=p_{i}$ present in the sample selected, and
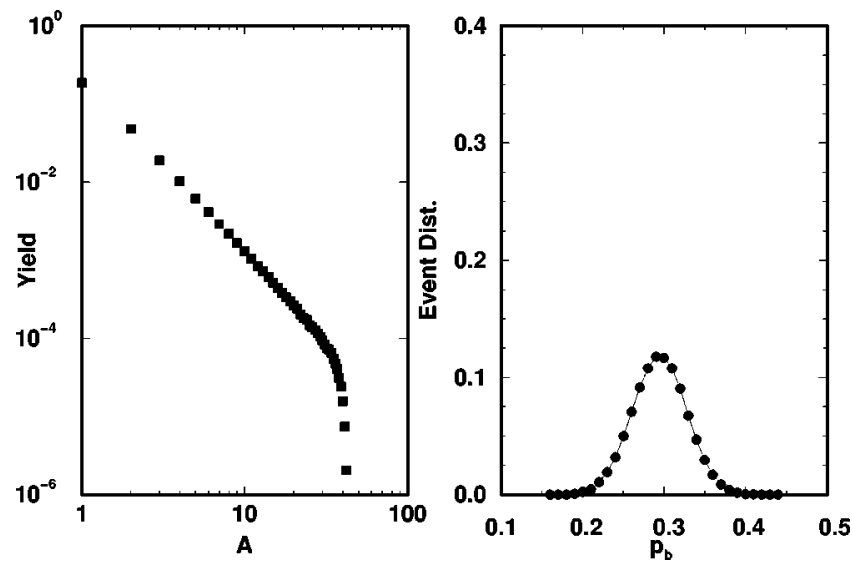

FIG. 2. Mass distribution (left panel) and distribution of breakup probabilities (right panel) of percolation events contained in the central rectangle of previous figure.
TABLE I. The values of $\tau$ vary substantially for all 12 regions of Fig. 1.

\begin{tabular}{lcccccc}
\hline \hline Region & $\mathrm{A}$ & $\mathrm{B}$ & $\mathrm{C}$ & $\mathrm{D}$ & $\mathrm{E}$ & $\mathrm{F}$ \\
$\tau$ & 2.8 & 2.6 & 2.2 & 2.1 & 2.9 & 2.5 \\
Region & $\mathrm{G}$ & $\mathrm{H}$ & $\mathrm{I}$ & $\mathrm{J}$ & $\mathrm{K}$ & $\mathrm{L}$ \\
$\tau$ & 2.3 & 2.2 & 2.6 & 2.75 & 2.3 & 2.3 \\
\hline
\end{tabular}

$\Sigma_{i} n\left(p_{i}\right)$ the total number of events in the rectangle. The right panel of Fig. 2 shows that this distribution is very broad indeed, and that the mix contains events coming from very different bond activation probabilities.

To see if the $p_{b}$ variation is related to the relative position of the event in the Campi plot, we further divide the rectangle in 12 regions, as shown in the insert of Fig. 1, and calculate the bond probability distribution for each of these regions. Table I shows the values of $\tau$ thus obtained, and Fig. 3 some typical probability distributions. As we can see the mixing is always high and the values of $\tau$ vary substantially independent of the relative position in the Campi plot. The selection rule used appears not to reduce the mixing of noncritical events.

But the effect of the cut is even more troublesome. If a Campi plot is produced using only $p_{c}$ (i.e., with the probability that produces the critical power-law mass distribution), the cut introduces large deviations in the expected critical mass distribution. The value of $\tau$ obtained in such a case of critical but cut-selected events is again too big: $\tau=2.3$. Apparently, the cut produces a biased exclusion of certain components of the mass distributions which are needed to yield the correct power-law distribution.

In summary, the usage of a cut not only does not help to reduce the existing (and unavoidable) mixing of critical and noncritical events, but it also disturbs the mass distribution in an uncontrollable way. Many other cuts were also explored, and although some cases led to better $\tau$ 's, specially those increasing the upper limit of the rectangle, none yielded $p_{b}$

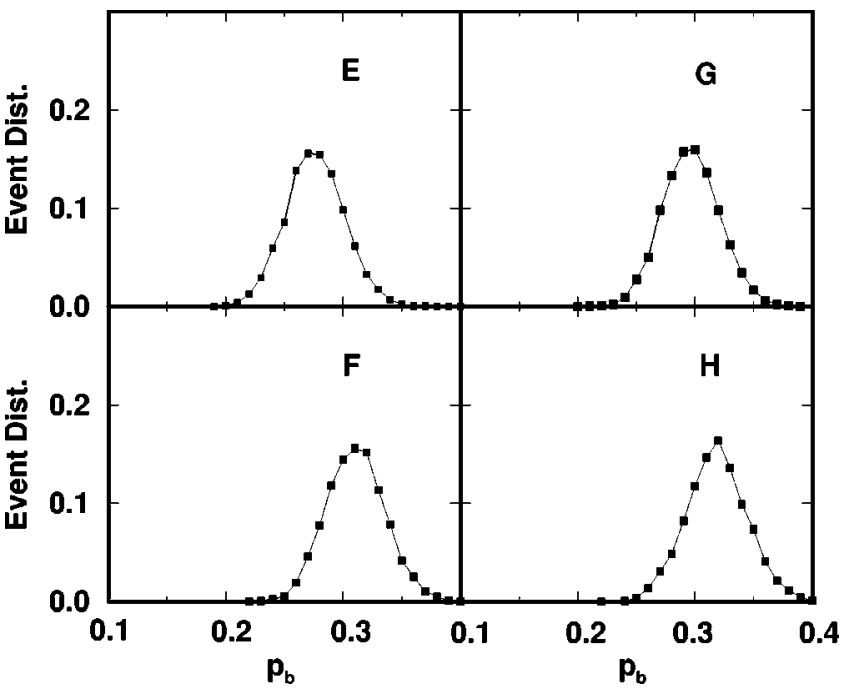

FIG. 3. Typical bond probability distribution in some of the regions of the central rectangle. 

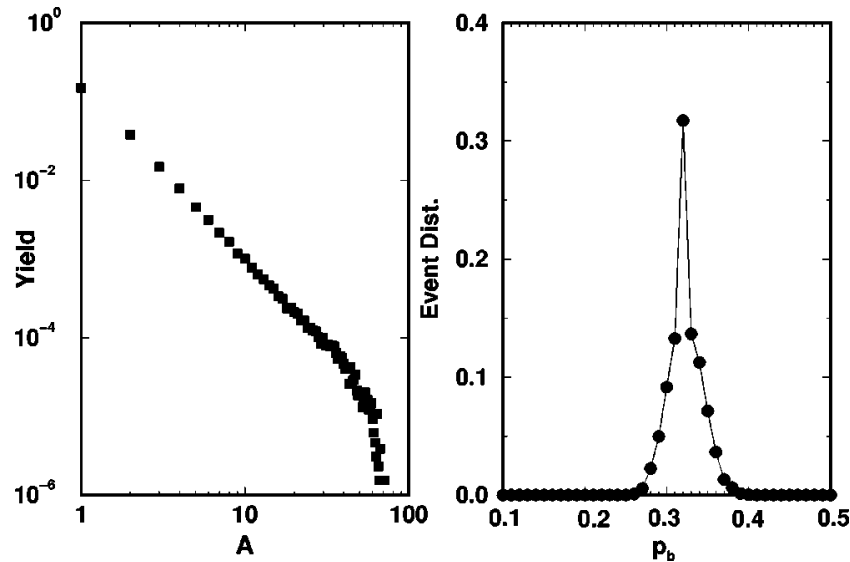

FIG. 4. Left panel: mass distribution and the power-law fit from the bin with the best fit. The critical exponent is $\tau=2.19 \pm 0.02$. Right panel: distribution of bond-activation probabilities $P_{i}$.

distributions with $p_{c}$ higher than $20 \%$. (For other deficiencies of the Campi plot see Ref. [18].)

In view of this, an event selection method other than the used cuts must be implemented. The ultimate goal is to be able to extract critical breakups from a mixture of events, and since these critical events produce a power-law mass distribution at a preferred critical multiplicity, an approach based on multiplicity selection, as proposed in Ref. [3], seems to be more promising. The underlying idea is to reduce the volume of data (mixed events) with a minimum loss of critical information by classifying the events in multiplicity bins, and then finding the multiplicity bin which produces the best one-parameter fit, i.e., the critical multiplicity. The procedure can be expected to work as Elliot and co-workers have managed to show that the total multiplicity is monotonically connected to the bond-activation probabilities in the case of percolation [19]. (We have also verified this for our calculations and have observed a small multiplicity deviation.)

The results of this multiplicity binning and fitting procedure is shown in Fig. 4 for the percolation calculations presented before. The left panel of Fig. 4 shows the mass distribution from the bin that produced the best power law fit, and the power-law curve that resulted from the fit. The value obtained for the critical exponents is $\tau=2.19 \pm 0.02$ in exact agreement with the true value.

To understand why this procedure yields better results for $\tau$, the right panel of Fig. 4 shows the distribution of bondactivation probabilities $P_{i}$. As evidenced by the sharp peak

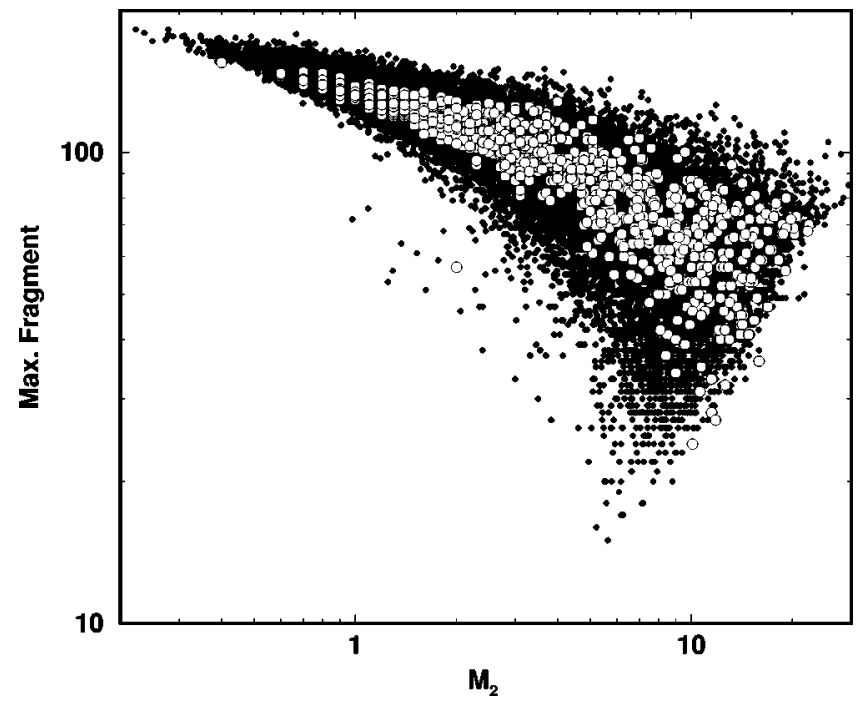

FIG. 5. Campi plot with all the critical events from the percolation data (dark circles), and the events in the critical multiplicity bin (white circles). Critical events are distributed over the whole region, including outside the elbow region. Events in the multiplicity bin sampled by the bin-and-fit rule also have a wide spread.

at $p_{c}$, the bin that produced the closest critical multiplicity, contains a narrower distribution of $P_{i}$ with a much reduced mixing of noncritical events than other bins.

To underline this last result, Fig. 5 shows a Campi plot with all the critical events produced in the percolation exercise (dark circles) at $p_{b}=p_{c}$, along with those events sampled in by the bin-and-fit procedure (white circles) for $p_{b}=p_{c}$. Just as in the wide distribution of the critical events explains the failure of the cut selection, the wide coverage of the sampled events explains the goodness of the bin-and-fit rule.

In conclusion, the selection of events using cuts on the Campi scatter plot does not reduce the relative number of noncritical events and perturbs the mass distribution in an undesirable way. Sorting events by multiplicity bins and selecting the one that gives the best one-parameter fit, not only seems to give much better results, i.e., events with a much higher concentration of critical events, but it is also a well defined methodology.

This work was supported by the Universidad de Buenos Aires (Grant No. TW98) and the National Science Foundation (Grant No. PHY-96-00038). J.A.L. acknowledges the hospitality of the Universidad de Buenos Aires, and C.O.D. that of the University of Texas at El Paso.
[1] J. A. López and C. O. Dorso, Phase Transitions in Nuclear Matter (World Scientific, Singapore, 2000).

[2] M. Bruno, A. Bonasera, C. O. Dorso, and J. P. Mastinu, Nuevo Cimento 2, 1 (2000).

[3] J.B. Elliot et al., Phys. Rev. C 62, 064603 (2000).

[4] J. Pochodzalla et al., Phys. Rev. Lett. 75, 1040 (1995).

[5] A. Strachan and C.O. Dorso, Phys. Rev. C 59, 285 (1999).
[6] F. Gulminelli and P. Chomaz, Phys. Rev. Lett. 82, 1402 (1999).

[7] M. D’Agostino et al., Nucl. Phys. A650, 329 (1999).

[8] M. D’Agostino et al., Phys. Lett. B 473, 219 (2000).

[9] A. Chernomoretz, M. Ison, S. Ortiz, and C. O. Dorso, Criticality in Nuclear Fragmentation (unpublished).

[10] X. Campi, J. Phys. C 48, 2 (1987).

[11] D. Stauffer and A. Aharony, Introduction to Percolation 
Theory (Taylor \& Francis, London, 1992).

[12] M. Belkacem, V. Latora, and A. Bonasera, Phys. Rev. C 52, 271 (1995).

[13] W. Bauer et al., Proceedings of the 7th High Energy Heavy Ion Study (GSI, Darmstadt, Germany, 1984), p. 701.

[14] W. Bauer et al., Phys. Lett. 150B, 53 (1985).

[15] W. Bauer et al., Nucl. Phys. A452, 699 (1986).
[16] W. Bauer et al., Phys. Rev. C 38, 1297 (1988).

[17] X. Campi and J. Desbois, Proceedings of the 23rd Bormio Conference (Ric. Sci. Educ. Perm., Milano, 1985), p. 497.

[18] A. Barrañón, C. O. Dorso, and J. A. López, Rev. Mex. Fis. (to be published).

[19] J.B. Elliot et al., Phys. Rev. C 55, 1319 (1997). 